\title{
Development of biosensor based on imaging ellipsometry and biomedical applications
}

\author{
G. Jin ${ }^{\text {a,* }}$, Y.H. Meng ${ }^{\text {a }}$, L. Liu ${ }^{\text {a }}$, Y. Niu ${ }^{\text {a }}$, S. Chen ${ }^{\text {a }}$, Q. Cai ${ }^{\text {b }, ~ T . J . ~ J i a n g ~}{ }^{b}$ \\ ${ }^{a}$ NML, Institute of Mechanics, Chinese Academy of Sciences, \#15 Bei-si-huan west Rd., Beijing 100190, China \\ b Institute of Biophysics, Chinese Academy of Sciences, Beijing 100101, China
}

\section{A R T I C L E I N F O}

Available online 27 December 2010

\section{Keywords:}

Imaging ellipsometry

Biosensor

Protein microarray

Total internal reflection

\begin{abstract}
A B S T R A C T
So far, combined with a microfluidic reactor array system, an engineering system of biosensor based on imaging ellipsometry is installed for biomedical applications, such as antibody screen, hepatitis B markers detection, cancer markers spectrum and virus recognition, etc. Furthermore, the biosensor in total internal reflection (TIR) mode has be improved by a spectroscopic light, optimization settings of polarization and low noise CCD which brings an obvious improvement of 10 time increase in the sensitivity and SNR, and 50 times lower concentration in the detection limit with a throughput of 48 independent channels and the time resolution of $0.04 \mathrm{~S}$.
\end{abstract}

(c) 2010 Elsevier B.V. All rights reserved.

\section{Introduction}

It has been 15 years since the concept of biosensor based on imaging ellipsometry (BIE) was proposed $[1,2]$. During the period, many previous contributions to its development, such as a biosensor based on off-null sampling method was developed for the visualization and quantitative measurement of the biomolecular interaction [1], which just formed a prototype of the biosensor concept. An affinity biochip with a matrix of 900 targets for detection affinity bonding showed the high-throughput ability of the biosensor with imaging ellipsometry [3]. An automatic immunosensor based on imaging ellipsometry and micro-fluidic system was developed to quantify the thickness distribution of protein layers or detect protein concentration in solution [4,5]. Furthermore, a micro-fluidic reactor array combined with total internal reflection imaging ellipsometry (TIRIE) was developed [6] for biomolecular interaction real-time detection. And the optimization method of null \& off-null ellipsometry is developed with emphasis on the improvement of sample thickness resolution $[7,8]$.

So far, the biosensor based on imaging ellipsometry has been developed into an automatic technique for protein analysis with merits of label-free, quantitative, high throughput and real-time analysis for protein interaction process, etc. Its principle, methodology, related technique units, biosensor system and previous works are reviewed in ICSE4 [4].

\footnotetext{
* Corresponding author. Tel.: +8610 82544138; fax: + 861082544138 E-mail address: gajin@imech.ac.cn (G. Jin).
}

An improved engineering system has been installed recent years. It includes an imaging ellipsometry system as a reader for sampling and a microfluidic reactor array for biomolecular interaction controlled by an operation system for an automatic performance. There is a database containing all information of the biosensor for biomedical applications.

\section{Engineering model}

Considering the dispersive effect of the microarray to improve imaging spatial resolution, we recently develops a spectroscopic imaging ellipsometry (SIE) $[9,10]$ system which has the lateral resolution of optical imaging ellipsometry with various wavelengths.

The diagram of the SIE setup is shown in Fig. 1. A 150-W Xenon short arc lamp (OSRAM, Germany) operated by a supply (Newport, U.S.) with light ripple less than $0.5 \%$ regulation accuracy is used to provide a stable outgoing flux with continuous spectrum from $\operatorname{IR}(1700 \mathrm{~nm})$ to UV $(190 \mathrm{~nm})$. In order to adequately utilize the light flux, an achromatic convergent lens set with a focal length of $200 \mathrm{~mm}$ and a diameter of $50 \mathrm{~mm}$ is used to converge the light beam into the entrance slit of a mechanical scanning grating monochromator (7-Star, China) with a focal length of $150 \mathrm{~mm}$ and an $\mathrm{f}$-number of $1 / 4.5$. The monochromator controlled by a computer provides a spectrum of $260-1600 \mathrm{~nm}$ with adjustable entrance and an exit for the control of light intensity and bandwidth, respectively. A glass optical fiber bound (Chunhui, China) with a spectrum of $380-1300 \mathrm{~nm}$ and a diameter of $1.0 \mathrm{~mm}$ is mounted flexibly to transfer the incident light from the exit of the monochromator to the focus point of the second achromatic convergent lens set. It collimates the single wavelength light into an expanded parallel beam 


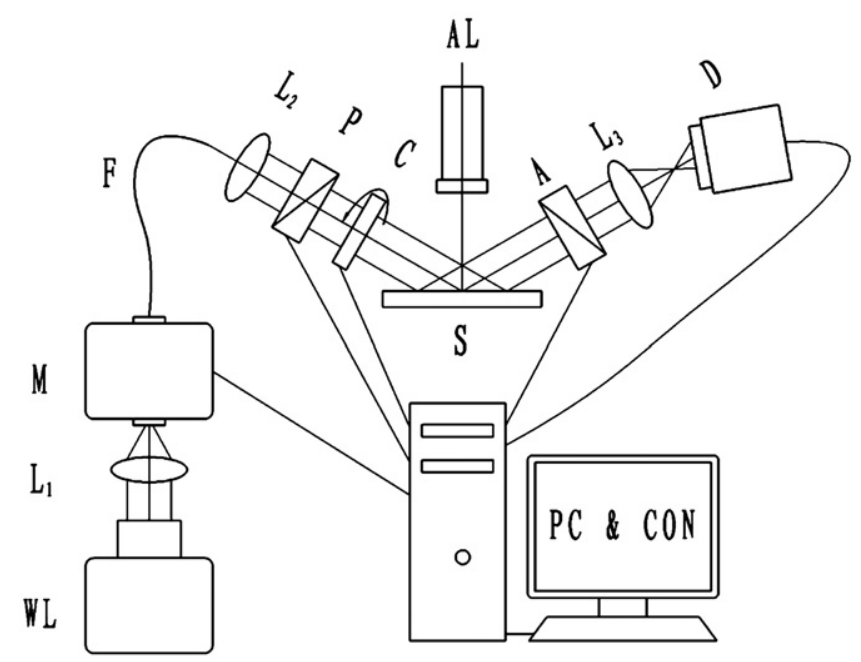

Fig. 1. the diagram of spectroscopic imaging ellipsometry setup, WL: Xe lamp; $\mathrm{L}_{1}, \mathrm{~L}_{2}$ : achromatic lens set; $M$ : scanning grating monochromator; F: optical fiber; P: polarizer; C: compensator; S: specimen; A: analyzer; AL: alignment telescope; $\mathrm{L}_{3}$ : achromatic imaging lens set; D: CCD camera; PC \& CON: computer and controller.

with a diameter of $25 \mathrm{~mm}$ for imaging ellipsometry at an angle of incidence.

Two Glan-Taylor prisms (MELLES GRIOT, USA) with a spectral range of $250-2300 \mathrm{~nm}$ and an extinction ratio of $10^{-5}$ are used as the polarizer and the analyzer, respectively. A mica retardation plate (MELLES GRIOT, USA) with a spectral range of $400-700 \mathrm{~nm}$ is used as a compensator. Its phase retardation between fast and slow axis is near $90^{\circ}$, which is calibrated by a commercial V-VASE ellipsometer (J.A.Woollam, USA) and fitted by the Cauchy model. In order to adjust the azimuth with respect to the plane of incidence, the polarizer, compensator, and analyzer are fixed in hollow annular rotation stages driven by a servo motor (Newport, US) with a maximum speed of $40^{\circ} \%$ $\mathrm{s}$ and an absolute accuracy of $0.015^{\circ}$. The azimuth of the polarizing parts is calibrated by a self-calibrating method [11]. The specimen is fixed in the specimen stage by a vacuum pump with a linear-motordriven free piston (NITTO KOHKI, Japan) and aligned by an optical self-collimated telescope (Micro-Radian, US).

An achromatic imaging lens set (Zeiss, Germany) with a focal length of $50 \mathrm{~mm}$ and an $\mathrm{f}$-number of 1.4 is used to image the specimen onto a scientific B/W video CCD camera (SONY, Japan). The camera features a corresponding spectrum range of $400-900 \mathrm{~nm}$, sensitive area of $768 \times 576$ pixels and $6.0 \times 4.96 \mathrm{~mm}^{2}$ (single pixel size of $6.5 \times 6.25 \mu \mathrm{m}^{2}$ ). The electronic gain is fixed for quantitative photonic intensity measurement. The sampling speed is 30 frames/S at maximum. The nonlinearity of the CCD camera is better than $1 \%$. The image signal is inputted into an image grab board (Matrox, Canada) inserted in the computer for analog-to-digital (A/D) conversion and the digital image processing and storage. The ellipsometric images are recorded in 8-bit (0-255) grayscale format. Image averaging method available is used to improve the signal-tonoise ratio [12].

In order to carry out the quantitative measurement for the biosensor purpose, the SIE can work in two kinds of mode upon requirement.

The first mode called null and off-null mode [2] is suitable for realtime applications of biomolecular fast interactions. In this mode, the surface mass concentration is measured by the well-defined relationship between the surface mass concentration and the grayscale value of image [8]. The sampling speed is up to 25 images per second due to no moving elements. A calibration for the layer thickness or the surface mass concentration is needed with a known sample for a quantitative detection with the biosensor. Normally the differential signal between the substrate and binding biomolecule layers is used as the sensing signal for biomolecular interaction, so the contrast of image is corresponding to the sensitivity of the sampling with imaging ellipsometry.

The second mode is a rotating compensator sampling method [13] which is suitable for some biomolecular slow interaction or ex-situ detection since it has the mechanical moving compensator, but it may quantitatively provide ellipsometric parameters in images. In order to get the information of the protein microarray, the following procedure is performed. Firstly, the azimuth of the compensator is rotated step by step with equal angle interval, while an ellipsometric image in grayscale is captured at each azimuth of the compensator as each sampling point, where the grayscale value of each pixel corresponds to a micro-area on the specimen. Secondly, the Fourier analysis is employed to get Fourier coefficients and then ellipsometric parameters $(\Psi, \Delta)$ are deduced. Thirdly, an appropriate physical model is used to describe the layered structure of a pattern on substrate. Furthermore, a data fitting procedure is performed to get the effective thickness or refractive index of the bio-layer in the pattern. Finally, a relationship [8] between the surface mass concentration of the bio-molecule and the fitted effective thickness $\&$ refractive index is used to get the information of the pattern area of interest.

The SIE system is automatically controlled by a computer including motion control hardware and a home-made operation software for image acquisition and data process. The major motion functions include the initialization of self-calibration and azimuth setting, the magnification adjustment according to the size of the specimen and the lateral resolution, the incident angle adjustment, the various magnification, the auto-focusing, the wavelength scanning, and the positioning for the feedback of the location of moving components.

The image capture is upon requirements such as the number of frames, the time interval, the average of multi-image, and the image processing including the choice of an interested area, the readout of the grayscale value in the area of interest and the image transfer to 3D, and so on.

Data processing mainly includes Fourier analysis to obtain Fourier coefficients, ellipsometric angles $\Psi(\lambda, \mathrm{x}$, and $\mathrm{y})$ and $\Delta(\lambda, \mathrm{x}$, and $\mathrm{y})$ of each micro-area of the specimen corresponding to a pixel in an image, $\mathrm{x}$ and $\mathrm{y}$ are perpendicular coordinates of image and the deduction for the properties of the specimen such as the thickness and the distribution of thickness and refraction index.

The SIE is used to determine the surface mass concentration. The advantage of the SIE is to achieve the surface mass concentration distribution on a solid substrate with optical lateral resolution in the order of micron upon the magnification. The present setup for images of $768 \times 576$ pixels spends the sampling time less than $8 \mathrm{~s}$ at each wavelength in the spectrum range of $400-700 \mathrm{~nm}$. Further fitting analysis with the model air- $\mathrm{SiO}_{2}-\mathrm{Si}$ indicates that the thickness resolution is approximately in the order of $0.1 \mathrm{~nm}$.

Here we should indicate that the rotating compensator sampling approach with detected photo number varying in a small range compared with a rotating polarizer (or analyzer) sampling in conventional ellipsometer, so it is quite suitable for the imaging ellipsometer to obtain ellipsometric parameters in image since the detector of CCD has a much smaller intensity dynamic range than a normal photo-detector or photomultiplier, but the accuracy of ellipsometric parameters is less than that by a rotating polarizer (or analyzer) sampling.

The biosensor may work in two modes: the ex-situ performance with independent microarray reactor system and in-situ performance in total internal reflection mode with a coupled microfluidic reactor array of 48 independent channels at a present mode for real-time detection. The microfluidic reactor array is used for various specific bindings between biomolecules with properties of label-free, multisample parallel detection. Its operation is simple even for simultaneous multi-channel analysis [14]. For most clinic applications tested, 
some detection results are good enough to answer if targets of interests exist or not and the dynamics of biomolecular interaction are not necessary to measure. In this way, the ex-situ performance with an independent microfluidic reactor array system for biomolecule detection is suitable. The detection result can be read by imaging ellipsometry without influence of liquid background. However, for other purpose of interests in biomolecular interaction process in situ, the detection with biosensor in situ mode is needed. It could mimic the solution environment in vivo and observe, measure and analyze the biological reaction process in real-time. Biosensor based on total internal reflection known as TIRIE biosensor is a suitable mode. The microfluidic reactor array is coupled with the optical sampling system by a prism. The incident light beam totally reflects at the interface between the substrate (gold layer on glass plate) and the tested solution when the incident angle is larger than a critical angle. The evanescent wave is used as optical probe to detect the protein adsorption and interaction process at the interface of solution/gold. The information of biomolecular interaction is obtained by analyzing the real-time process. It is important to know properties of the biomolecule and screening target molecule in medicine, etc. Meanwhile, TIRIE biosensor may have high sensitivity since it applies an evanescent wave for optical detection. It's a powerful tool in the detection of biomolecule weak-affinity interaction and clinic tests with a high sensitivity, but the influence coming from the solution background has to be considered.

\subsection{Improvement for TIRIE biosensor}

The sensitivity and flexibility of the biosensor is very important for practical applications. The sensitivity depends not only upon the resolution of imaging ellipsometry but also upon the bio-system of ligand-receptor on the microarray that is the bioactivity and its act is related to the ligand screen to choose the best among ligands upon their bioactivity, ligand immobilization, unspecific blocking and interaction conditions, etc. The flexibility mainly depends on the mechanical, electrical, informatics and biological control. The preliminary system which is a $8 \times 6$ biomolecule reactor-array developed as a promising technique for parallel protein test is introduced somewhere previously $[6,15]$. The sensitivity and the detection limit of the preliminary biosensor system are quite limited.

In order to improve the performance of the TIRIE biosensor, three optimization approaches are brought in the methodology. Besides considering the dispersion of materials used in the sensing surface of the biosensor, the spectroscopic light source applied instead of the single wavelength would provide an optimized dispersive choice. The optical settings of polarization components are optimized for maximum sensitivity [16,17]. Otherwise, a low noise CCD detector is equipped to improve the SNR (ratio of signal to noise) and imaging quality of the biosensor system so that an obvious improvement of 10 times in the sensitivity and 50 times lower concentration in the detection limit has been obtained [18].

The polarization setting results in an obvious improvement in the biosensor sensitivity which is discussed in detail somewhere else [19]. It is verified that polarization setting plays an important role in the biosensor sampling system when the angle of incidence, the setting of the wavelength and the optical parameters of sample are fixed. It shows that the image contrast varied with the azimuth setting of the polarizer and analyzer and optimal settings corresponding to the highest image contrast could be found.

Generally, improvement in the sensitivity and the detection limit could be achieved by increasing the SNR. In TIRIE biosensor, the noise sources include noises from temperature fluctuation in an imaging device, a light source and from the flow disturbance in micro-fluidic reactor, etc. A cool CCD (Andor DU937N-BV) with low noise function and a time resolution of $0.04 \mathrm{~S}$ is equipped as the imaging device of TIRIE biosensor instead of the scientific B/W video CCD camera (SONY,
Japan). The cool CCD with low noise (Andor DU937N-BV, read noise $10.3 \mathrm{e}$ at $2.5 \mathrm{MHz}$, dark current $0.0005 \mathrm{e}-/ \mathrm{pixel} / \mathrm{s}$ at $-80^{\circ} \mathrm{C}$ ) as the imaging device can improve SNR more than 10 times.

\subsection{Database}

According to features of the biosensor, it can be widely used in detecting and monitoring biomolecular interaction, especially in the field of biomedicine. For example, more than a hundred kinds of proteins recognized as diseases markers are classified by their natures. Thus, a biosensor database may play an important role for potential users. The database based on network hosts includes some of the representative results of BIE experiment data which can be classified as antigen-antibody, hormone, ligand-receptor, and virus, etc. The schematic view of the database focusing on tumor markers spectrum detection is shown in Fig. 2. The contents of database are derived from author's latest own work including quantitative detection of CD146, the antibody screening of poison ricin [20] and cooperative research groups, such as Suzhou Institute of Nano-tech and Nano-bionics(SINANO), Chinese Academy of Science (CAS) [8]; Institute of Biophysics (IBP), CAS; Institute of Microcirculation, Chinese Academy of Medical Science [21], etc. It is composed of the data from samples and experiments which is about 100 items for users. Taking the item CA15-3 [21] as a target example, the database is composed of primary data (such as the molecule name, mass, source, reservation condition, references about CA15-3) and the experiment's data (the ligand-anti CA15-3, calibration curve, experiment conditions such as the microarray substrate, ligand immobilization, operation process, solution delivery, reaction time and results). In addition, the database could be expanded by our group and other users just to complement further targets in the corresponding items of the database. It's designed to provide an efficient and authoritative platform for BIE data indexing and sharing.

\section{Some biomedical applications}

Some representative biomedical application results recent years are reviewed here, such as the quantitative detection of tumor marker CD146, the antibody screening of poison ricin [20], the detection of five hepatitis B markers [22], various virus recognition [23,24] and tumor markers' spectrum presented first time, etc.

\subsection{Quantitative detection of CD146}

CD146 glycoprotein belonging to cell adhesion molecules is considered to be a novel target on endothelial cell involved in tumor angiogenesis. BIE performed in null and off-null modes is used for CD146 detection as a trial by the following steps. Firstly, CD146 antibody as ligand is immobilized on modified silicon substrate by Protein G. Then, CD146 detection is performed and its calibration curve is established for the need of quantitative detection. Finally, 18 serum samples are tested quantitatively, and their results are compared with ELISA's. The sensitivity for CD146 detection achieves the order of ng/ml and the relationship between BIE signal y (grayscale value) and CD146 concentration $\mathrm{x}(\mathrm{ng} / \mathrm{ml})$ is $\mathrm{y}=3.3 \ln (\mathrm{x})+91.3$ shown in Fig. 3 . Compared with ELISA's, the majority of results are in agreement, and the results of two approaches have significant statistic relevance shown in Fig. 4.

\subsection{Ricin antibody screening [20]}

Ricin found in castor beans is considered to be one of the most potent plant toxins and its estimated oral lethal dose is less than $20 \mathrm{mg} / \mathrm{kg}$ body weight in humans. Its antibody can identify and neutralize ricin fast and efficiently and is of great significance for diagnosis and treatment to those who are exposed to ricin poison. BIE 


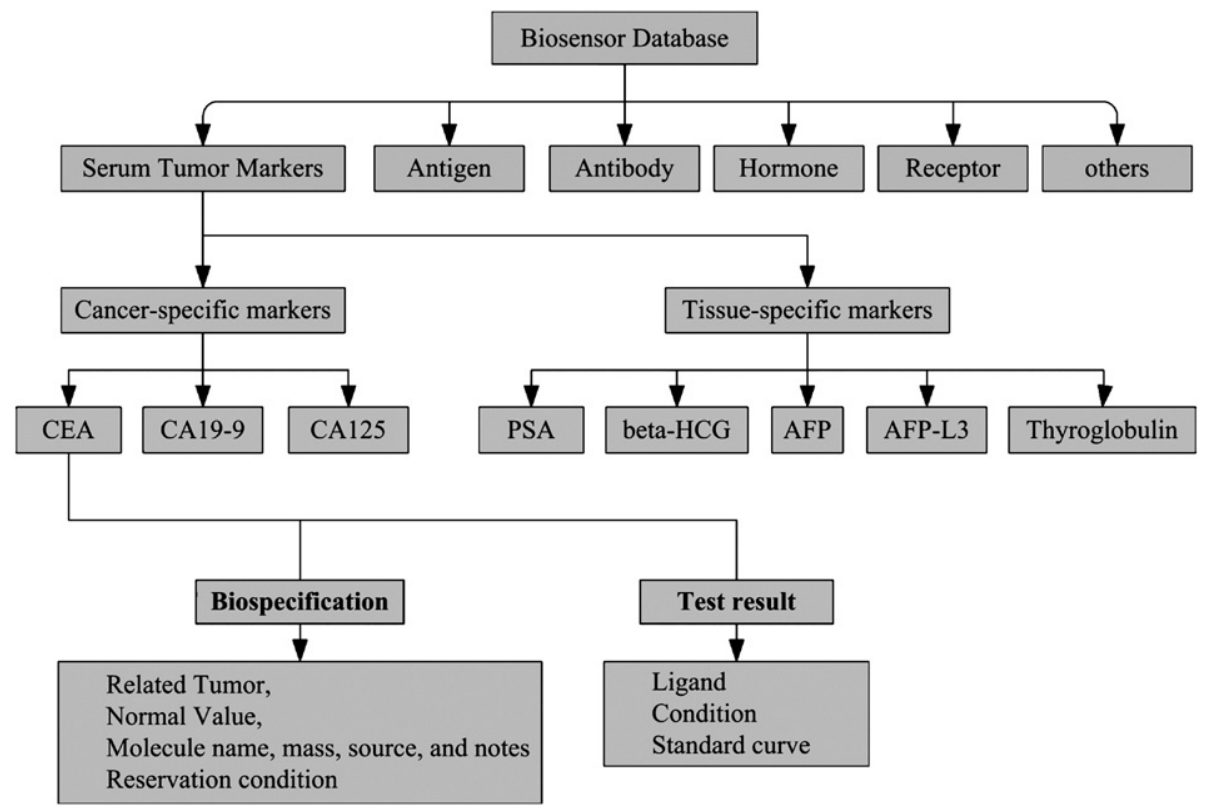

Fig. 2. Database of the biosensor.

set in null and off-null mode is developed to be an antibody screening platform for ricin antibodies comparison and evaluation. Five kinds of ricin antibody named 7G7, pVHHS1, 5S1R, VHHS1 and S1R are immobilized in an array format on the surface modified with succinic anhydride, respectively. Ricin ranging from $0.5 \mathrm{ng} / \mathrm{ml}$ to $5 \mu \mathrm{g} / \mathrm{ml}$ (much lower than the lowest oral lethal dose) dissolved in PBS solution is measured for the limit of detection (LOD) values of the five kinds of antibody, while abrin as a similarity to ricin is set as a negative control. The results corresponding to the array valued in grayscale are shown in Table 1. The LOD values of 7G7, pVHHS1, 5S1R, VHHS1 and S1R are $5 \mathrm{ng} / \mathrm{ml}, 500 \mathrm{ng} / \mathrm{ml}, 1 \mathrm{ng} / \mathrm{ml}, 1 \mu \mathrm{g} / \mathrm{ml}$ and $10 \mathrm{ng} /$ $\mathrm{ml}$ detected in the indicated concentration of Ricin, respectively, which are basically in accordance to their characteristics and ELISA results obtained by our copartner that their LOD values are in the order of $\mathrm{ng} / \mathrm{ml}, \mu \mathrm{g} / \mathrm{ml}, \mathrm{ng} / \mathrm{ml}, \mu \mathrm{g} / \mathrm{ml}$ and $\mathrm{ng} / \mathrm{ml}$, respectively. LOD values of antibody 7G7, 5S1R and S1R present in the order of $\mathrm{ng} / \mathrm{ml}$ more efficient than other two kinds of antibody and the 5S1R is chosen as the best for its lowest LOD value. 7G7, 5S1R and S1R may play potential roles in the clinical diagnosis and therapy.

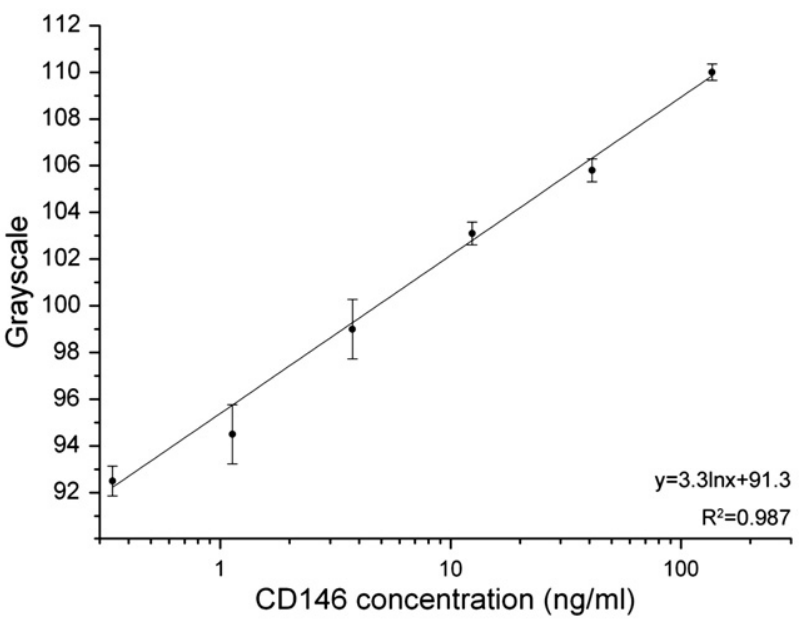

Fig. 3. The calibration curve for quantitative detection of CD146.

\subsection{Detection of five HBV markers [22]}

Five markers of the hepatitis B virus (HBV) including the hepatitis B surface antigen (HBsAg), the hepatitis B surface antibody (HBsAb), hepatitis B e antigen ( $\mathrm{HBeAg}$ ), hepatitis B e antibody (HBeAb) and hepatitis $B$ core antibody ( $\mathrm{HBCAb}$ ) are a group of general markers used in the monitoring of HBV infection. 169 patient sera are detected by BIE for the purpose of clinical diagnosis, which includes the ligand screening, the determination of the sensitivity, the cutoff values ( $\mathrm{CoVs}$ ) and the comparison with ELISA test. The receiver operating characteristic (ROC) curve method [22] which has been widely used in the field of clinic to evaluate efficiency of detection method is introduced to deduce the CoVs of the five markers. BIE can simultaneously detect five markers of several samples in one microarray during only $1 \mathrm{~h}$ and its results have been compared with ELISA in good agreement. The sensitivity of $1 \mathrm{ng} / \mathrm{ml}$ and $1 \mathrm{IU} / \mathrm{ml}$ for $\mathrm{HBsAg}$ and HBsAb has been achieved, respectively (shown in Figs. 5

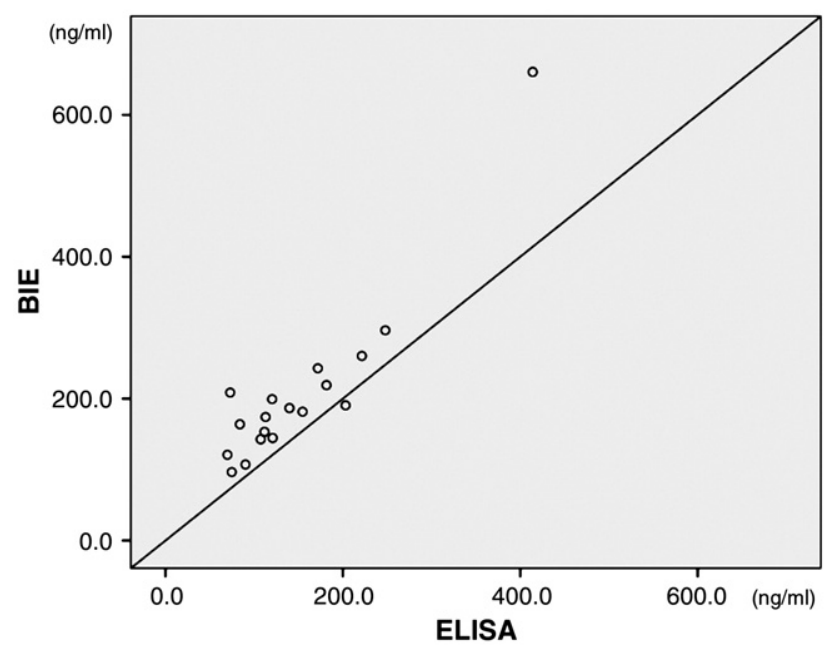

Fig. 4. Comparison between BIE and ELISA for CD146 detection of 18 patient sera. The detection results of 18 patient sera measured by BIE and ELISA are plotted in the same figure, and then are performed by the correlation analysis [27]. Pearson correlation coefficient [27] is 0.923 which means the results of two methods are significant at the level of 0.01 
Table 1

Detection of ricin in the array with acquired results in gray-scale value [20].

\begin{tabular}{lllrrr}
\hline & $7 G 7$ & pVHHS1 & 5S1R & VHHS1 & \multicolumn{1}{c}{ S1R } \\
\hline Blank control & 106.8 & 101.2 & 98.0 & 95.3 & 95.1 \\
Ricin & 118.1 & 108.9 & 114.1 & 100.5 & 104.9 \\
Negative control & 107.5 & 102.0 & 99.1 & 96.1 & 96.3 \\
\hline
\end{tabular}

and 6). The CoVs of the five markers: HBsAg, HBsAb, HBeAg, HBeAb and $\mathrm{HBCAb}$ are deduced to: $15 \%, 18 \%, 15 \%, 20 \%$ and $15 \%$, respectively, which are the percentage over the values of corresponding to negative control (national reference negative samples).

\subsection{Detection of phage M13KO7 [24]}

Phages are estimated to be the most widely distributed and diverse entities in all reservoirs populated by bacterial hosts. Phage M13KO7 is a kind of virus that resides in Escherichia coli. Although it has no infection on humans and animals, it functions as a model to develop BIE as an approach to detect virus directly since Phage M13KO7 size and structure is similar to virus infecting human. Different phage M13KO7 samples, such as natural and purified samples and different batches of samples with various concentrations, are directly detected by BIE. Interaction between avidin and biotin is adopted for immobilization of mouse biotin-anti-M13 monoclonal antibody (bio-GP3) against phage M13KO7 with a model of micro-fluidic for a microarray. The surface with saturated layer of bio-GP3 is incubated in a solution containing phage M13KO7 which is caught by bio-GP3 with the interaction between phage M13KO7 and bio-GP3 detected by BIE (Shown in Fig. 7) and determined by AFM (shown in Fig. 8). The lowest detection limit of BIE is $109 \mathrm{pfu} / \mathrm{ml}$ (pfu/ml is a unit used in phage which means the number of plaque forming units per unit volume). By comparing BIE with AFM, both BIE and AFM are label-free detection method, but BIE has properties of rapid test, multiplex analysis, quantitative detection and convenient operation.

a

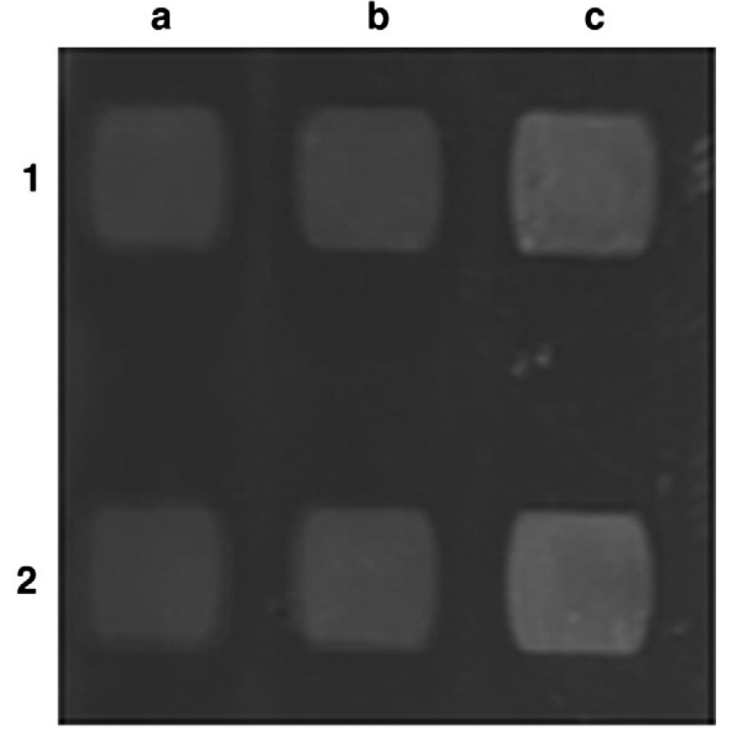

Fig. 5. Sensitivity in detecting marker HBsAg. HBsAb as ligand is immobilized on 6 areas, and then 6 areas are blocked with HBsAg national reference negative sample. Following this, HBsAg national positive reference samples with $1 \mathrm{ng} / \mathrm{ml}$ are detected on area "c1" and "c2". HBsAg national reference negative samples as negative control are detected on area "b1" and "b2". PBS solution with $0.1 \%$ Tween-20 (PBST) as blank control is added to the rest area "a1" and "b1" [22]. a b C

3

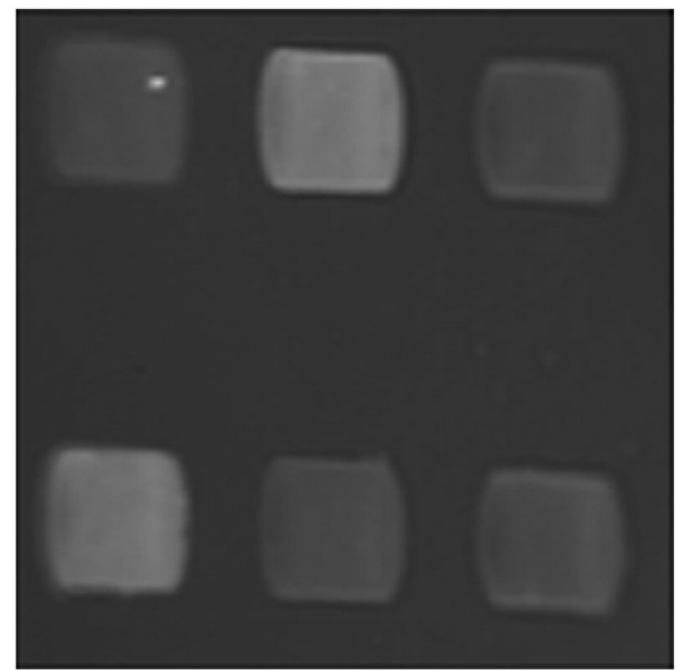

Fig. 6. Sensitivity in detecting marker HBsAb. HBsAg as ligand is immobilized on 6 areas, and then 6 areas are blocked with HBsAb national reference negative sample. Following this, HBsAb national positive reference samples with $1 \mathrm{IU} / \mathrm{ml}$ are detected on area "b1" and "c2". HBsAg national reference negative samples as negative control are detected on area "a1" and "b2". PBST as blank control is added to the rest area "c1" and "c2" [22].

\subsection{Detection of Avian influenza virus [23]}

Avian influenza virus (AIV) has been causing global concern as a potential pandemic threat to human life. As a typical animal virus, it is detected by BIE for developing a quick AIV detection tool. A sensing surface with multi-kinds of antibody against AIV in a patterned array is constructed as a probe in order to capture and collect AIV as a result of the specific identification and affinity interaction between antibodies and virus. The BIE result shows the virus detection (shown in Fig. 9 and Table 2). The subtype H5N1 with high pathogenicity is detected with a sensitivity of $2.56 \times 10^{-3} \mathrm{TCID} / \mathrm{ml}$ (tissue culture infectious dose, TCID), which is confirmed by a scanning near-field optical microscopy (SNOM) performing in a

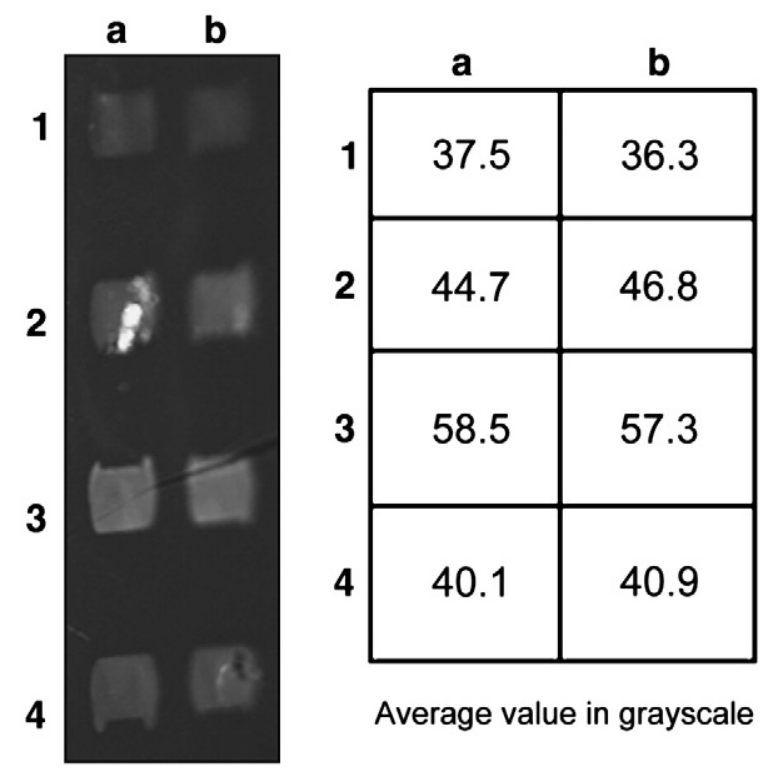

Fig. 7. The BIE image verifies specific interaction of bio-GP3 and phage M13KO7. In the image: Avidin is firstly immobilized on all areas, and then bio-GP3 is immobilized on areas "a2, a3, a4, b2, b3, and b4". In the detection, areas "a2 and b2" are added with PBST as control; areas "a3 and b3" and "a4 and b4" are used to detect phage M13KO7 and negative sample (SARS), respectively [24]. 

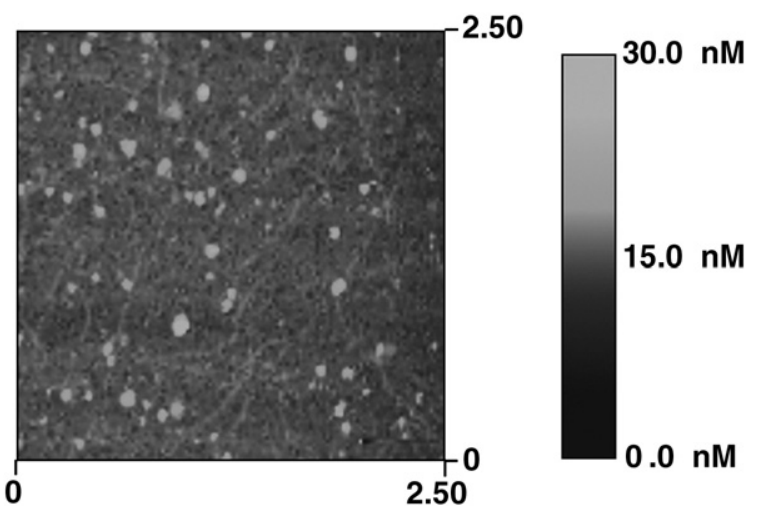

Fig. 8. AFM image of $2.5 \mu \mathrm{m}$ scanning distance on the area dedicated for phage detection. The original and overall configuration of phage M13KO7 on the mica surface is presented in this figure. The particles in the image are considered to be contaminants in culture solution of phage, while the phage M13KO7 is a long filament (its length is about $1 \mu \mathrm{m}$, height is about $4.42 \mathrm{~nm}$ and width is about $39.21 \mathrm{~nm}$ ) [24].

shear force mode and the image of AIV captured on sensing surface is shown in Fig. 10.

\subsection{Detection of tumor markers spectrum}

Tumor markers directly corresponding to the presence of a tumor open a window in the tumor stage diagnosis. However, a detection result of single tumor marker is only considered as a reference other than the dominant evidence to confirm the diagnosis for its drawback in specificity. Alpha-fetoprotein (AFP), alpha-L-fucosidase (AFU) and Ferritin are common tumor markers. We try to use these in the detection of liver cancer which is the most popular tumor in China. The three tumor markers are designed to measure with BIE simultaneously as a trial to explore the tumor marker spectrum feasibility in clinic in order to increase the diagnostic specificity [25].

Firstly, with the ligand screening as above to determine optimized concentrations of AFP antibody, AFU antibody and Ferritin antibody as ligand, protein A is assembled on the carboxyl modified silicon surface to realize oriented immobilization of antibody. Standard tumor markers of AFP, AFU and Ferritin in several concentrations are used to install a calibration curve for BIE quantitative test. The calibration

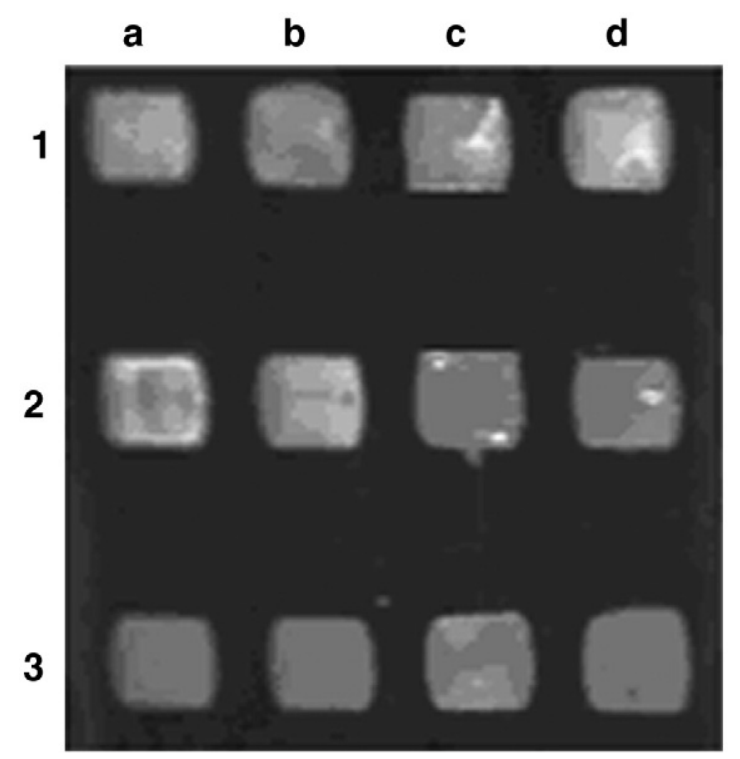

Fig. 9. Detection of AIV samples by BIE, antibody ScFv is bound on the a and b columns; the antibody 4A4 in ascites is bound on the c and d columns; H5N1 is detected in the 1 row; H9N2 is detected in the 2 row; the 3 row is used as a ligand control [23].
Table 2

Detection of AIV samples in grayscale value [23].

\begin{tabular}{lllll}
\hline & ScFv & ScFv & 4 A4 & 4 A4 \\
\hline H5N1 & 169.5 & 154.6 & 191.3 & 193.4 \\
H9N2 & 156.7 & 170.0 & 137.9 & 137.8 \\
Control & 130.1 & 130.2 & 129.3 & 135.0 \\
\hline
\end{tabular}

curves of AFP, AFU and Ferritin are $\mathrm{y}=13.4 \lg (\mathrm{x})+79.7$ (detection range is from $1 \mathrm{ng} / \mathrm{ml}$ to $64 \mathrm{ng} / \mathrm{ml}$ ), $\mathrm{y}=24.6 \lg (\mathrm{x})+88.1$ (detection range is from $1 \mathrm{U} / \mathrm{l}$ to $64 \mathrm{U} / \mathrm{l}$ ) and $\mathrm{y}=14.5 \lg (\mathrm{x})+76.8$ (detection range is from $5 \mathrm{ng} / \mathrm{ml}$ to $160 \mathrm{ng} / \mathrm{ml}$ ), respectively, while y is the BIE signal in grayscale value and $\mathrm{x}$ the tumor marker concentration in $\mathrm{ng} / \mathrm{ml}$ or $\mathrm{U} / \mathrm{l}$. 32 normal sera and 24 liver cancer patient sera are quantitatively measured (shown in Fig. 11).

ROC curve for single marker detection and Logistic regression for joint markers test is introduced to analyze results to make an evaluation of BIE performance in diagnosis of liver cancer. The area under curve (AUC) which gives the relationship between the sensitivity and specificity for an independent test is $0.827,0.782$ and 0.380 for AFP, AFU and Ferritin, respectively (shown in Fig. 12). However, AUC increases to 0.884 with a joint detection of AFP and AFU, while the Ferritin is much less related to the both for liver cancer diagnosis. It shows that the joint detection of tumor markers of AFP and AFU may bring more feasibility than their individual detections for clinic purpose.

\subsection{Quantitative detection of interaction among vesicular membrane fusion proteins}

A rapid detection and identification of vesicular protein-protein interactions is essential not only for the understanding of vesicle trafficking, but also for the understanding of the system-level organization of cellular structure, biological information processing and molecular mechanism [26]. Here we use BIE to evaluate interactions among vesicular proteins Sec22, Ykt6, Sso1 and Sso2. The four vesicular proteins as ligands are immobilized to form four columns over 4 cells in an array, and then the four vesicular proteins are adopted to add in rows over 4 cells, respectively and array $4 \times 4$ couples of the vesicular proteins for their cross interactions. With the statistic standard of P-value [27], the evaluation result shows that the interaction between Ykt6 and Sso2 is significant $(\mathrm{P}=0.0084$, P-value equal to or less than 0.05 is positive). These vesicular proteins belong

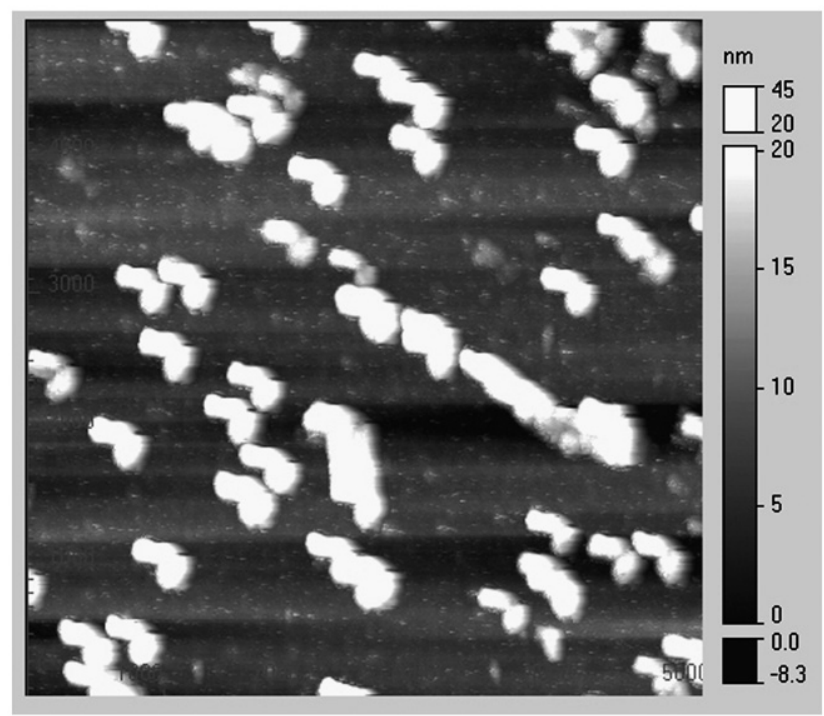

Fig. 10. Images of SNOM in a shear force mode for H5N1 detection by BIE [23]. 


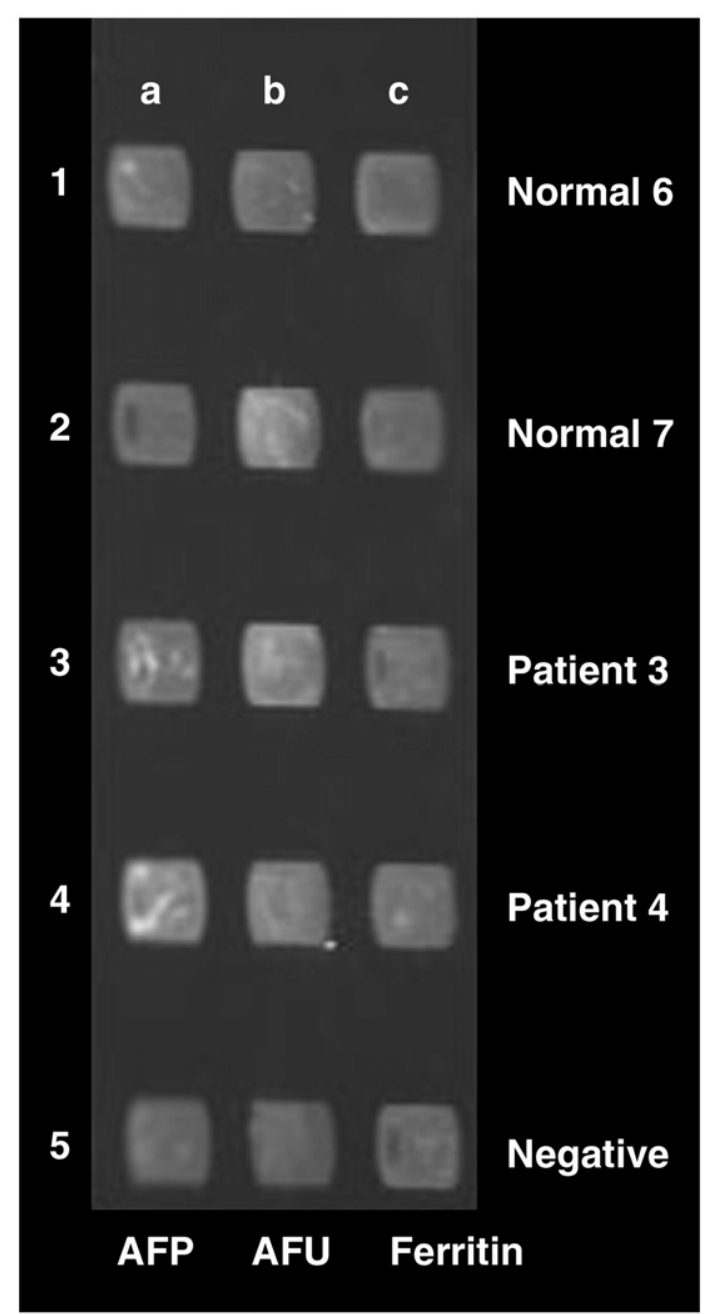

Fig. 11. Detection of tumor markers' spectrum composed of AFP, AFU and Ferritin, column a, b and c is immobilized by anti-AFP, anti-AFU and anti-Ferritin as ligand; while Row 1, 2, 3, 4 and 5 is reacted with 2 normal sera, 2 patient sera and negative control, respectively.

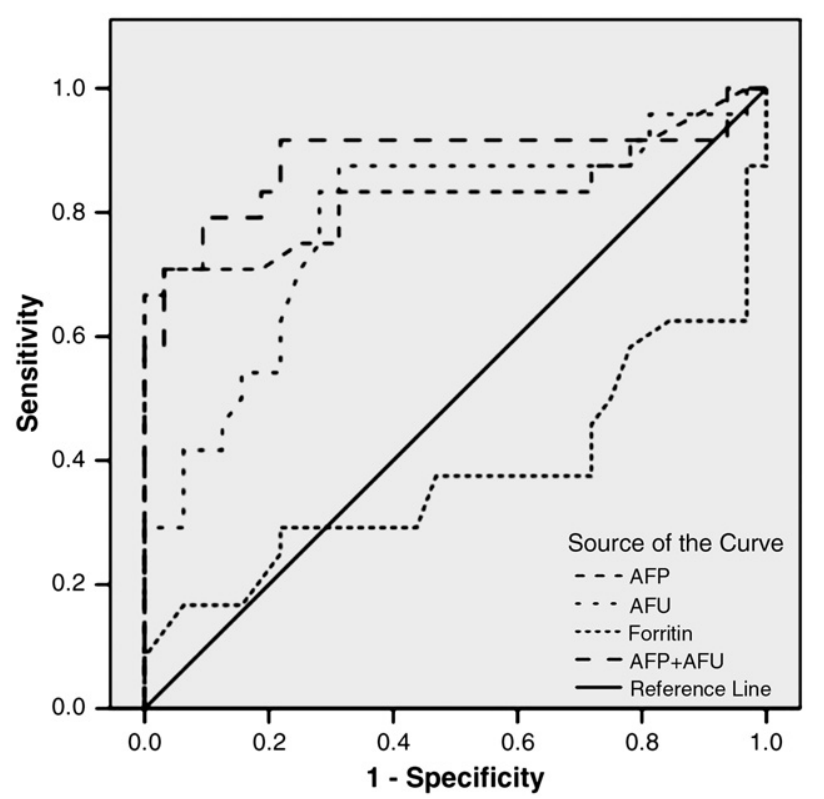

Fig. 12. ROC curve analysis of tumor markers of AFP, AFU, Ferritin and joint detection of AFP and AFU. to SNEAR (Soluble N-Ethylmaleimide-Sensitive Factor Attachment Protein Receptor) family. Ykt6 and Sso2 interaction plays an important role in vesicular trafficking. These vesicular proteins assemble into coiled-coil tetramers to promote membrane fusion [28]. The detection result in vitro by BIE is consistent with the result of yeast two-hybrid assays (Y2H) at a domain level in vivo [29].

\subsection{Vesicles adsorption process visualization}

Vesicles are used as a model for cell membrane due to its lipid bilayer structure. Its adsorption behavior is significant to study the properties of cell membrane [30,31]. TIRIE biosensor performed in null and off-null modes is applied to visualize vesicles (made of 1, 2Dioleoyl-sn-glycero-3-phosphocholine) non-specific adsorption and desorption on poly-L-lysine modified gold surface shown in Fig. 13.

The 6th region of curve "a" and "b" in Fig. 13 is the vesicle nonspecific adsorption on the poly-L-lysine. The signal reaching to the maximum expressed in grayscale is about 900 in increase. In the 7th region, the solution is changed to the glucose solution with a $5 \mu \mathrm{l} / \mathrm{min}$ flux, the signal decreases since the unbound vesicles are rinsed by the glucose solution. When the flux increases to $10 \mu \mathrm{l} / \mathrm{min}$, the signal almost keeps the same since the flux is not large enough to remove more vesicles. Increasing the flux to $30 \mu \mathrm{l} / \mathrm{min}$, the signal in curve "a" and "b" decreases 300 in grayscale, but it keeps constant in curve "c". These demonstrate that vesicles are removed from the surface for the shear force destroys the non-specific adsorption between vesicles and poly-L-lysine. Increasing the flux again and it reaches to $40 \mu \mathrm{l} / \mathrm{min}$, the signal in curves "a", "b" and "c" decrease simultaneously for the polyL-lysine is wiped off from the surface.

To verify the result of TIRIE biosensor, the parallel experiment is operated with a phase contrast microscope (NIKON, TI-U, Japan). The results with TIRIE biosensor and the microscope are accordant. As evidence by the microscopic result, the process of the vesicle adsorption, desorption with the flow rate, as well as the poly-L-lysine layer destruction on the surface could be visualized by TIRIE biosensor. Generally, the size of the vesicles is in the order of micron so that it is an attempt of the TIRIE biosensor in micron target detection.

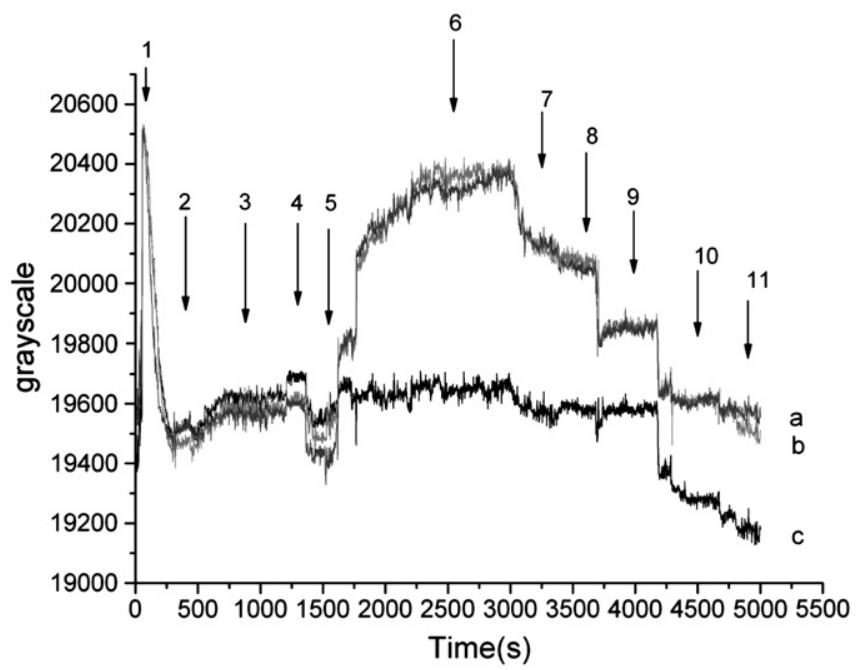

Fig. 13. Curves "a" and "b" correspond to the vesicle adsorption and desorption. Curve "c" is reference. Region 1 - activation with N-hydroxy-droxysuccinimide and 1-(3dimethylaminopropyl)-3-ethylcarbodimide hydrochloride; 2 - pure water rinsing; 3 poly-L-lysine with concentration of 1:100 immobilization; 4 - pure water rinsing; 5 glucose solution with $\mathrm{pH}$ 10; 6 - vesicles in glucose solution with $\mathrm{pH} 10$ binding with poly-L-lysine; 7 - glucose solution rinsing with flux of $5 \mu \mathrm{l} / \mathrm{min}$.; 8 - glucose solution rinsing with $10 \mu \mathrm{l} / \mathrm{min}$.; 9 - glucose solution rinsing with $30 \mu \mathrm{l} / \mathrm{min}$.; 10 - glucose solution rinsing with $40 \mu \mathrm{l} / \mathrm{min}$.; 11 - glucose solution rinsing with $45 \mu \mathrm{l} / \mathrm{min}$. 


\section{Summary}

An automatic engineering system of the biosensor has been installed with a spectroscopic imaging ellipsometer as a reader within the wavelength range of $400-700 \mathrm{~nm}$, which may be performed in both sampling modes of Null \& Off-null and rotating compensator for fast image sampling and images of ellipsometric parameters for further characterization. The biosensor may be performed in two operational modes that are either the ex-situ with an independent microfluidic reactor array for biomolecule detections or the in-situ run in total internal reflection mode with a coupled microfluidic reactor array for real-time measurement of biomolecular interactions. It has achieved in more biomedical applications, such as the cancer marker CD146 detection, Ricin antibody screening, the detection of five HBV markers, the detection of Avian influenza virus, and the detection of tumor marker spectrum, etc. With a recent improvement with an optimization of wavelength and polarization settings as well as the use of a cool CCD, the sensitivity has increased one order and the detection limit for HBsAb and HBsAg 50 times better than before. It maintains several significant advantages: non-contact and label-free. Non-contact ensures the detection without disturbance to samples, while label-free guarantees the results with less false positive. It is demonstrated in image so its detection quality can be directly observed and evaluated in real-time. Moreover, it can be operated in parallel and high throughput analysis so that multiple detections of numerous samples can be carried out simultaneously. So far, it's achieved $1 \mathrm{ng} / \mathrm{ml}$ for $\mathrm{HBsAg}$, the sensitivity basically meets the demand for most clinical purpose, so that it brings a bright potential for further biomedical applications.

\section{Acknowledgements}

The Chinese Academy of Sciences YZ0629, KJCX2-YW-M03 and KJCX2-YW-M04; the National Basic Research Program of China 2009CB320300; and the National High Technology Research Development Program (863) of China 2008AA02Z419, NNSFC 20805053 are acknowledged for their financial supports. The authors gratefully acknowledge K. C. Wong Education Foundation, Hong Kong. One of authors would like to gratefully acknowledge financial supports from China Postdoctoral Science Foundation funded project.

\section{References}

[1] G. Jin, P. Tengvall, I. Lundstrom, H. Arwin, Anal. Biochem. 232 (1995) 69.

[2] G. Jin, R. Jansson, H. Arwin, Rev. Sci. Instrum. 67 (1996) 2930.

[3] P. Van Gerwen, W. Laureyn, A. Campitelli, P. Jacobs, P. Detemple, K. Baert, W. Sansen, R. Mertens, J. Micromech. Microeng. 10 (2000) N1.

[4] G. Jin, Phys. Status Solidi A 205 (2008) 810.

[5] Z.H. Wang, G. Jin, Anal. Chem. 75 (2003) 6119.

[6] Y.Y. Chen, Z.H. Wang, Y.H. Meng, G. Jin, Int. J. Nanotechnol. 4 (2007) 171.

[7] H. Arwin, M. Poksinski, K. Johansen, Appl. Opt. 43 (2004) 3028.

[8] Y.Y. Chen, Y.H. Meng, G. Jin, Appl. Opt. 46 (2007) 8475.

[9] Y.H. Meng, S. Chen, G. Jin, Chin. Opt. Lett. Suppl. 8 (2010) 114.

[10] Y.H. Meng, Y.Y. Chen, C. Qi, L. Liu, G. Jin, Phys. Status Solidi C 5 (2008) 1050.

[11] I. An, J.A. Zapien, C. Chen, A.S. Ferlauto, A.S. Lawrence, R.W. Collins, Thin Solid Films 455 (2004) 132.

[12] Y.H. Meng, G. Jin, Opt. Precis. Eng. 8 (2000) 316.

[13] R. Kleim, L. Kuntzler, A. Elghemmaz, J. Opt. Soc. Am. A Opt. Image Sci. Vis. 11 (1994) 2550.

[14] G. Jin, Z.H. Wang, Int. J. Nonlinear Sci. Numer. Simul. 3 (2002) 191.

[15] Z.H. Wang, Y.H. Meng, P.Q. Ying, C. Qi, G. Jin, Electrophoresis 27 (2006) 4078.

[16] G.L. Wang, H. Arwin, R. Jansson, Appl. Opt. 43 (2004) 2000.

[17] G.L. Wang, H. Arwin, R. Jansson, Appl. Opt. 42 (2003) 38.

[18] L. Liu, Y.Y. Chen, Y.H. Meng, S. Chen, G. Jin, 5th International Conference on Spectroscopic Ellipsometry, Albany, NY USA, 2010 (TSF-D-10-01047).

[19] L. Liu, Y. Niu, S. Chen, Y.H. Meng, H.W. Ma, G. Jin, Sci. China, Ser. G: Phys. Mech. Astron. 53 (2010) 1805.

[20] Y. Niu, J. Zhuang, L. Liu, X.Y. Yan, G. Jin, Thin Solid Films (2010), doi:10.1016/j. tsf.2010.12.054

[21] H.G. Zhang, C. Qi, Z.H. Wang, G. Jin, R.J. Xiu, Clin. Chem. 51 (2005) 1038.

[22] C. Qi, W. Zhu, Y. Niu, H.G. Zhang, G.Y. Zhu, Y.H. Meng, S. Chen, G. Jin, J. Viral Hepat. 16 (2009) 822.

[23] C. Qi, X.S. Tian, S. Chen, J.H. Yan, Z. Cao, K.G. Tian, G.F. Gao, G. Jin, Biosens. Bioelectron. 25 (2010) 1530.

[24] C. Qi, Y. Lin, J. Feng, Z.H. Wang, C.F. Zhu, Y.H. Meng, X.Y. Yan, L.J. Wan, G. Jin, Virus Res. 140 (2009) 79.

[25] H. Chen, C.M. Jiang, C. Yu, S. Zhang, B.H. Liu, J.L. Kong, Biosens. Bioelectron. 24 (2009) 3399.

[26] J.S. Bonifacino, B.S. Glick, Cell 116 (2004) 153

[27] D. Freedman, R. Pisani, R. Purves, A. Adhikari, Statistics, Norton \& Company Inc., New-York NY, 1991.

[28] D. Ungar, F.M. Hughson, Annu. Rev. Cell Dev. Biol. 19 (2003) 493.

[29] H. Zhang, J.A. Chen, Y.Q. Wang, L. Peng, X.X. Dong, Y. Lu, A.E. Keating, T.J. Jiang, J. Mol. Biol. 392 (2009) 228.

[30] K. Sengupta, L. Limozin, Phys. Rev. Lett. 104 (2010) 088101.

[31] D. Cuvelier, C. Vezy, A. Viallat, P. Bassereau, P. Nassoy, J. Phys. Condens. Matter 2004 (2004) S2427. 